\title{
Transcription factor AP-2 $\alpha$ negatively regulates thymic stromal lymphopoietin expression in respiratory syncytial virus infection
}

\author{
JIA HE* , QIAN CAO* , DAN-DAN FENG, JIA-HE CHEN and GUO-PING ZHOU \\ Department of Pediatrics, The First Affiliated Hospital, Nanjing Medical University, Nanjing, Jiangsu 210029, P.R. China
}

Received November 11, 2019; Accepted April 30, 2020

DOI: $10.3892 / \mathrm{mmr} .2020 .11192$

\begin{abstract}
Respiratory syncytial virus (RSV) infection enhances the cell-mediated immune responses of type 2 helper $\mathrm{T}$ cells and promotes the progression of allergic inflammation and asthma by producing thymic stromal lymphopoietin (TSLP), especially long isoform TSLP (lfTSLP). However, the role of short isoform TSLP (sfTSLP) in RSV infection remains to be elucidated. The present study was designed to demonstrate the role of both 1fTSLP and sfTSLP, as transcription regulators, in RSV infection. The expression of lfTSLP and sfTSLP in RSV-infected Beas-2B cells was analyzed. Activating protein 2 (AP-2) $\alpha$ was overexpressed or knocked down to detect the changes in sfTSLP and IfTSLP expression. Luciferase reporter plasmid and chromatin immunoprecipitation experiments demonstrated that AP- $2 \alpha$ bound to the sfTSLP promoter region. LfTSLP and sfTSLP increased while AP- $2 \alpha$ decreased in RSV-infected Beas-2B cells. In the Beas-2B cells, AP- $2 \alpha$ was found to negatively regulate the activity of the sfTSLP promoter and the mRNA level of SfTSLP. AP- $2 \alpha$ also negatively regulated the expression of lfTSLP at both the mRNA and protein levels. The results of the chromatin immunoprecipitation assay indicated that AP- $2 \alpha$ bound to the core promoter region of sfTSLP. These results confirmed that the transcription factor AP-2 $\alpha$ can repress the expression of IfTSLP and sfTSLP in bronchial epithelial cells in RSV infection.
\end{abstract}

\section{Introduction}

Respiratory syncytial virus (RSV) infection enhances the cell-mediated immune responses of type 2 helper $\mathrm{T}$ cells

Correspondence to: Dr Guo-Ping Zhou, Department of Pediatrics, The First Affiliated Hospital, Nanjing Medical University, 300 Guangzhou Road, Nanjing, Jiangsu 210029, P.R. China

E-mail: zhougp2019@126.com

*Contributed equally

Key words: thymic stromal lymphopoietin, respiratory syncytial virus, bronchial epithelial cells, activating protein $2 \alpha$ and promotes the progression of allergic inflammation and asthma by producing thymic stromal lymphopoietin (TSLP), especially long isoform TSLP (lfTSLP) $(1,2)$. TSLP, an interleukin (IL)-7-like cytokine first discovered in the supernatant of thymic stromal cell cultures in mice, derives from the epithelium and acts in the pathophysiology of autoimmune and allergic diseases, including atopic dermatitis, allergic conjunctivitis, allergic rhinitis and asthma (3-5). TSLP exists in two distinct isoforms, lfTSLP and short isoform TSLP (sfTSLP). lfTSLP has a sequence of 159 amino acids, while sfTSLP relies on a downstream in-frame start codon to generate a peptide of either 63 or 60 amino acids, which fully overlaps the amino-acid sequence of IfTSLP in the C-terminal region (6). sfTSLP, the main isoform in the steady state, demonstrates protective and antimicrobial activity (3). In contrast, IfTSLP overexpression can promote the process of inflammation. Concerted research has been performed to explore the expression and related biological functions of these two isoforms in diseases $(3,4,6)$.

Early-life viral respiratory tract infections can increase the risk of recurrent wheezing and asthma (7-9). Respiratory syncytial virus (RSV) is a major causative agent of lower respiratory infections in the pediatric population, especially in infants (10). RSV infection triggers type 2 helper T cell (Th2) immune response characterized by IL-4, IL-5 and IL-13 production. The resultant excessive mucus in the respiratory tract enhances the pulmonary inflammatory response, subsequently leading to asthma $(1,2,11)$. TSLP produced by epithelial cells in RSV infection can create a permissive environment for the differentiation of dendritic cells, $\mathrm{T}$ cells and congenital lymphocytes and the subsequent production of the Th2-type cytokines $(2,12)$. Current research is more focused on lfTSLP, rarely on $\operatorname{sfTSLP}(2,12,13)$.

Activating protein 2 (AP-2) $\alpha$, a transcription-suppressor, may reduce TSLP transcriptional activity. Rs2289276, a single-nucleotide polymorphism (SNP) located in 5' untranslated region of sfTSLP and the intron 2 of IfTSLP, potentially changes the affinity of AP- $2 \alpha$ between two alleles. The allele containing AP-2 $\alpha$ binding site acts in a protective manner (13). This means that AP- $2 \alpha$ may participate in the transcriptional regulation of TSLP in a non-inflammatory state.

The present study aimed to determine the role of sfTSLP in RSV infection, the association between the two isoforms of TSLP and the potential transcriptional mechanisms. The 
results identified that sfTSLP can be regulated by AP- $2 \alpha$, supporting the hypothesis AP-2 $\alpha$ deficiency in bronchial epithelial cells might promote sfTSLP and lfTSLP expression in RSV infection.

\section{Materials and methods}

Cell lines. African green monkey kidney epithelial cells (Vero), human bronchial epithelium cells (Beas-2B) and HeLa cells were provided by the American Type Culture Collection. Cells were maintained in Dulbecco's modified Eagle's medium (Nanjing KeyGen Biotech Co., Ltd.), containing $80 \mathrm{U} / \mathrm{ml}$ penicillin and $0.08 \mathrm{mg} / \mathrm{ml}$ streptomycin, supplemented with $10 \%$ heat-inactivated fetal bovine serum (FBS, Gibco; Thermo Fisher Scientific, Inc.) at $37^{\circ} \mathrm{C}$ and in a humidified atmosphere of $5 \% \mathrm{CO}_{2}$.

Reagents. Rabbit anti-GAPDH antibody (cat. no. ab181602), rabbit anti-transcription factor AP-2 $\alpha$ (cat. no. ab52222) and rabbit anti-TSLP antibody (cat. no. ab47943) were purchased from Abcam. ProteinTech Group, Inc. provided the goat anti-rabbit IgG (cat. no. SA00001-2).

$R S V$ preparation and experimental infection. Human RSV type A (strain A2) was kept in the laboratory of the Department of Pediatrics, The First Affiliated Hospital, Nanjing Medical University and had been used in previous studies (14). As previously described, the virus was expanded in DMEM containing 2\% FBS in Vero cells. When the cytopathic effect reached $80 \%$, the infected cells were frozen-thawed in the medium. The RSV was purified by centrifugation $(850 \mathrm{x} \mathrm{g}$, $10 \mathrm{~min}, 4^{\circ} \mathrm{C}$ ) and then stored at $-80^{\circ} \mathrm{C}$ for further investigation. The viral titer was determined by $50 \%$ tissue culture infective dose in the Reed-Muench method (15) and was expressed as multiplicities of infection (MOI). The Beas-2B cells were cultured in DMEM mixed with $2 \%$ FBS for $24 \mathrm{~h}$ and then infected with RSV for $48 \mathrm{~h}$ before harvest and assay.

AP-2 $\alpha$ overexpression and small interfering (si)RNA knockdown. Cells $\left(1 \times 10^{5}\right)$ were seeded in 12-well plates overnight for transfection. Following the manufacturer's protocol, transfection was carried out in Beas-2B cells using Lipofectamine ${ }^{\circledR} 3000$ (Invitrogen; Thermo Fisher Scientific, Inc.). The AP- $2 \alpha$ expression plasmid was supplied by Thermo Fisher Scientific, Inc., and the pcDNA3.1-basic vector was used as a control. The customized siRNA was synthesized by Guangzhou RiboBio Co., Ltd., with a target sequence of GAGGAAGATCTTTAAGAGA. The controlled siRNA was also synthesized by Guangzhou RiboBio Co., Ltd., with a target sequence of CGUAAACGGCCACAAGUUC. The transfection reaction was carried out in DMEM without FBS. The expression plasmid $(1,000 \mathrm{ng} / \mathrm{ml})$ or siRNA $(100 \mathrm{nM})$ were transfected into cells. The cell culture medium was replenished with the extract $16 \mathrm{~h}$ after transfection. The cells were harvested for mRNA analysis $24 \mathrm{~h}$ after transfection and for protein analysis $48 \mathrm{~h}$ after transfection.

Reverse transcription-quantitative $(R T-q)$ PCR. Cells $\left(1 \times 10^{5}\right)$ were seeded in 12-well plates and lysed by TRIzol ${ }^{\circledR}$ (Invitrogen; Thermo Fisher Scientific, Inc.) $24 \mathrm{~h}$ after transfection. Total
RNA was extracted and purified according to the manufacturer's protocol. The amount and purity of RNA were measured using a NanoDrop 2000 Spectrophotometer (Thermo Fisher Scientific, Inc.). RNA (1,000 ng) was reverse-transcribed into cDNA using the Prime Script RT Master Mix Perfect Real Time kit (Takara Biotechnology Co., Ltd.). Samples were measured on a LightCycler 40 II instrument (Roche Diagnostics) using SYBR Green technology (Takara Biotechnology Co., Ltd.) for RT-qPCR. The mRNA level was normalized based on the level of GAPDH mRNA and calculated into a $2^{-\Delta \Delta C q}$ value (16). DNA amplification was performed as follows: Initial denaturation at $95^{\circ} \mathrm{C}$ for $30 \mathrm{sec}$, followed by 50 cycles of amplification (denaturation at $95^{\circ} \mathrm{C}$ for $5 \mathrm{sec}$ and annealing at $60^{\circ} \mathrm{C}$ for $30 \mathrm{sec}$ ). Fluorescence data were collected during the annealing phase of the amplification. All experiments were repeated at least three times. The primers used were: AP- $2 \alpha$ sense, 5'-ATTGACCTA CAGTGCCCAGC-3' and antisense, 5'-TCCATGAAAATGCT TTGGAA-3'; sfTSLP sense, 5'-CCGCCTATGAGCAGC CAC-3' and antisense, 5'-CCTGAGTAGCATTTATCTGAG-3'; IfTSLP sense, 5'-ACCAGTGGGAAGGGCAACC-3' and antisense, 5'-CATTGTTTGGCTGAAGGCTTGT-3'; GAPDH sense, 5'-ATGACATCAAGAAGGTGGTG-3' and antisense, 5'-CATACCAGGAAATGAGCTTG-3'.

Western blot assay. Cells were collected on ice $48 \mathrm{~h}$ after transfection and lysed in $200 \mu 1$ of pre-iced lysis buffer containing 0.1 mM PMSF (Nanjing KeyGen Biotech Co., Ltd.). Total protein was determined using a BCA assay. Protein samples (40 $\mu \mathrm{g} / \mathrm{lane})$ were loaded onto $10 \%$ precast polyacrylamide gradient gels (Beyotime Institute of Biotechnology) and transferred to nitrocellulose membranes (Immobilon; EMD Millipore) for $150 \mathrm{~min}$. To block non-specific sites, they were then incubated in TBS-T saline $(0.25 \mathrm{M}$ Tris- $\mathrm{HCl} ; \mathrm{pH} 7.6$, $0.19 \mathrm{M} \mathrm{NaCl}, 0.1 \%$ Tween-20) in 5\% dried milk for $2 \mathrm{~h}$ at room temperature. Protein blots were incubated for $12-14 \mathrm{~h}$ at $4^{\circ} \mathrm{C}$ with primary antibodies against TSLP, AP- $2 \alpha$ and GAPDH (1:2,000; diluted with Primary Antibody Dilution Buffer purchased from Beyotime Institute of Biotechnology). Then, membranes were incubated for $1 \mathrm{~h}$ at room temperature with a goat anti-rabbit IgG secondary antibody $(1: 10,000$; diluted with Secondary Antibody Dilution Buffer purchased from Beyotime Institute of Biotechnology). Reactive proteins were visualized by enhanced chemiluminescence (Biosharp Life Sciences) and densitometry was performed using a Bio-Rad gel imager and Quantity One 1-D version 4.6 software (Bio-Rad Laboratories, Inc.).

Bioinformatics prediction and plasmid construction. The luciferase reporter gene was cloned at MIUI/XhoI sites in the pGL3-Basic vector. A series of 5'-flanking region of sfTSLP were inserted to pGL3-Basic as previously (17). JASPAR (http://jaspar.genereg.net/analysis) (18) was used to predict the AP- $2 \alpha$ transcriptional sites of the promoter 5'-flanking region. The plasmids containing the core promoter of sfTSLP (pGL-200/+25) and mutations of the AP- $2 \alpha$ binding sites on the sfTSLP core promoter region were synthesized directly by TSINGKE Biological Technology Co., Ltd. All the plasmids were verified by Sanger sequencing (provided by TSINGKE Biological Technology Co., Ltd.). 
A

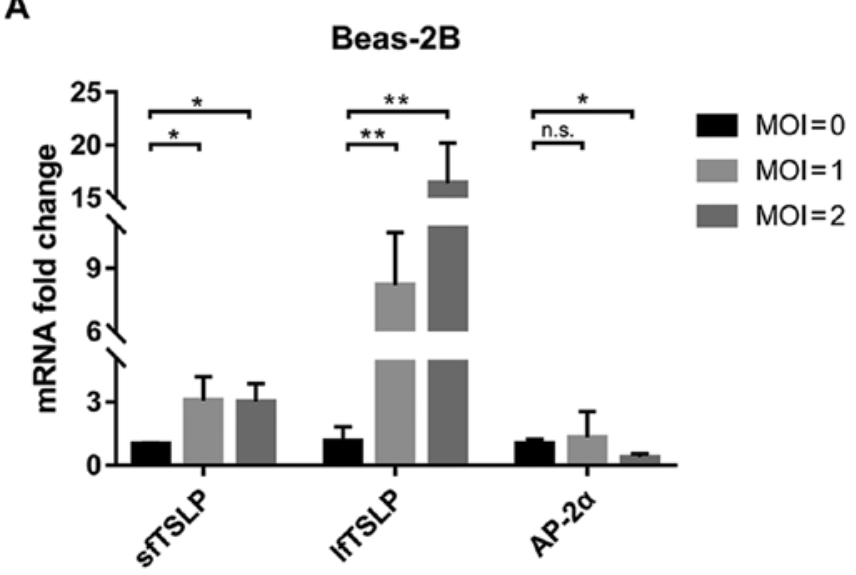

B

a

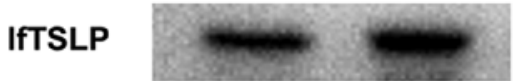

GAPDH

MOI

0

2

b

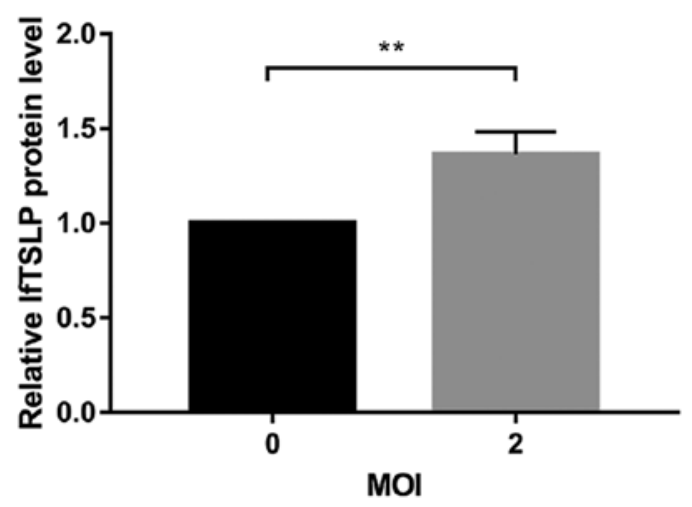

Figure 1. RSV infection upregulates TSLP expression in Beas-2B cells (A) Beas-2B cells were cultured for $48 \mathrm{~h}$ in the presence of RSV and lysed in TRIzol ${ }^{\circledR}$. Following total mRNA extraction, reverse transcription-quantitative PCR was performed using specific lfTSLP, sfTSLP and AP- $2 \alpha$ primers. (B) lfTSLP protein released from Beas-2B cells at $48 \mathrm{~h}$ of RSV $(\mathrm{MOI}=2)$ treatment was (a) measured by western blotting and (b) quantified. Representative data from $\geq 3$ independent experiments performed under similar conditions. ${ }^{*} \mathrm{P}<0.05,{ }^{* *} \mathrm{P}<0.01$ vs. the corresponding unstimulated controls; n.s., not significant; RSV, respiratory syncytial virus; TSLP, thymic stromal lymphopoietin; lf, long isoform; sf, short isoform; AP-2 $\alpha$, activating protein $2 \alpha$; MOI, multiplicity of infection.

Transient transfection and luciferase assay. For characterizing the reporter cell lines, $1 \times 10^{4} \mathrm{HeLa}$ cells were plated in 96-well plates overnight before transfection for luciferase assay. Transfection mixtures were prepared in OptiMEM I Reduced Serum Medium (Gibco; Thermo Fisher Scientific, Inc.) using Lipofectamine ${ }^{\circledR} 3000$ (Invitrogen; Thermo Fisher Scientific, Inc.). The AP- $2 \alpha$ expression plasmid (100 ng) or siRNA $(100 \mathrm{nM})$ was co-transfected into HeLa cells together with $100 \mathrm{ng}$ of luciferase reporter plasmids driven by wild-type or mutated sfTSLP promoter. Then, $4 \mathrm{ng}$ of the
pRL-TK plasmid (Promega Corporation) was co-transfected as a control plasmid to maintain the total DNA amount. Cells were stimulated $24 \mathrm{~h}$ after transfection and lysed in $30 \mu \mathrm{l}$ of lysis buffer (Promega Corporation). The Dual Reporter Assay System (Promega Corporation) and TD-20/20 Turner Designs Luminometer were used for measuring luciferase activity. Samples were analyzed in triplicate in each experiment and each experiment was repeated $\geq 3$ times.

Chromatin immunoprecipitation (ChIP) assay. According to the manufacturer's instructions, $2 \mu \mathrm{g}$ of anti-AP- $2 \alpha$, anti-Histone $\mathrm{H} 3$ antibody and control $\mathrm{IgG}$ antibody were analyzed in ChIP assay using the ChIP-IT kit (cat. no. 53008; Active Motif, Inc.) as described previously (14). The products were subjected to PCR amplification and further amplified by RT-qPCR with the following promoter-specific primers: sense, 5'-GGGAACGTTGTTAGGGGCA-3' and antisense, 5'-GGGGAACACAAGTCGAGAGT-3'. PCR products were resolved on a $2 \%$ agarose gel together with $100 \mathrm{bp}$ DNA ladder (Takara Biotechnology Co., Ltd.). Input DNA samples from the sonicated nuclear lysates and DNA samples immunoprecipitated with antibodies against IgG were used as positive or negative controls. All experiments were repeated three times.

Statistical analysis. Results are expressed as the mean \pm standard deviation of the mean. Student's t-test and Welch's ANOVA with a Tamhane post hoc test were performed using SPSS 22.0 (IBM Corp.) and GraphPad Prism 7 (GraphPad Software, Inc.). $\mathrm{P}<0.05$ was considered to indicate a statistically significant difference.

\section{Results}

RSV infection increases TSLP expression and decreases $A P-2 \alpha$ expression in Beas $-2 B$ cells. The present study first examined the effect of RSV infection on lfTSLP mRNA expression, finding that the mRNA and protein levels of lfTSLP were significantly increased in Beas-2B cells following RSV infection for $48 \mathrm{~h}$ (Fig. 1). Compared with the uninfected controls, a 16-fold increase in lfTSLP mRNA expression and a 3-fold increase in sfTSLP mRNA expression were detected in RSV-treated cells (MOI=2) for $48 \mathrm{~h}$ (Fig. 1A). It was also revealed that the level of AP- $2 \alpha$ mRNA expression was decreased by $62 \%$ (Fig. 1A). Western blotting also confirmed that IfTSLP expression was significantly increased after RSV infection (MOI=2; Fig. 1B).

$A P-2 \alpha$ regulates the expression of both lfTSLP and SfTSLP. The AP- $2 \alpha$ overexpression vector and siRNA AP- $2 \alpha$ were transfected into Beas-2B cells to examine the effect of AP- $2 \alpha$ on TSLP expression. The transfection efficacy of the overexpression vector was validated at the mRNA (Fig. 2A-c) and protein level (Fig. 2B-a and b). The transfection efficacy of the siRNA AP-2 $\alpha$ was validated at the mRNA (Fig. 2A-c) and protein level (Fig. 2C-a and b). Using RT-qPCR, it was found that the mRNA levels of both lfTSLP and sfTSLP were significantly decreased with the overexpression of AP- $2 \alpha$ (Fig. 2A-a and b). By contrast, significantly increased mRNA levels of IfTSLP and sfTSLP were observed in the AP- $2 \alpha$ knockdown cells (Fig. 2A-a and b). Western blotting also confirmed that 
A

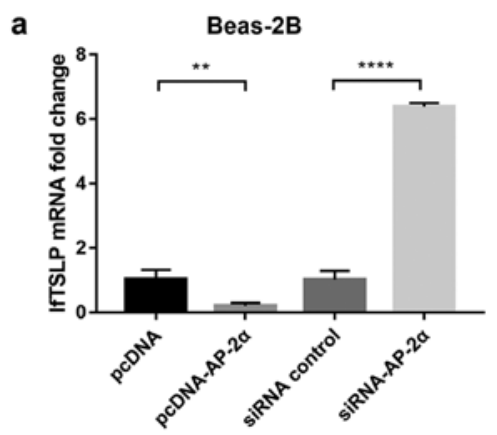

C

B
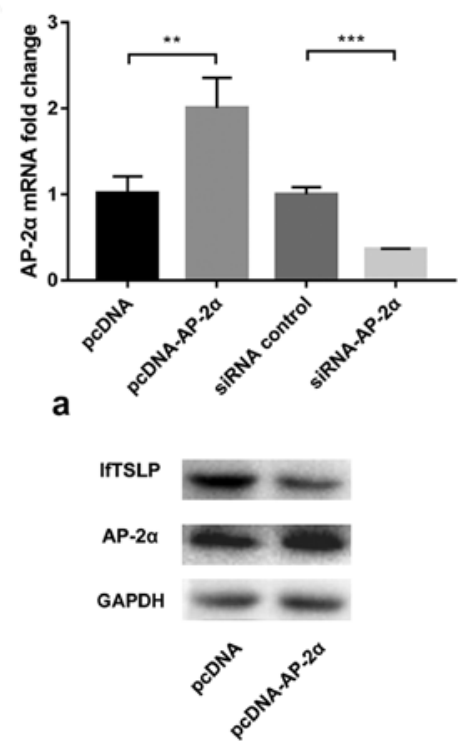

C

a

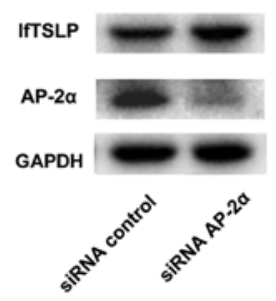

b

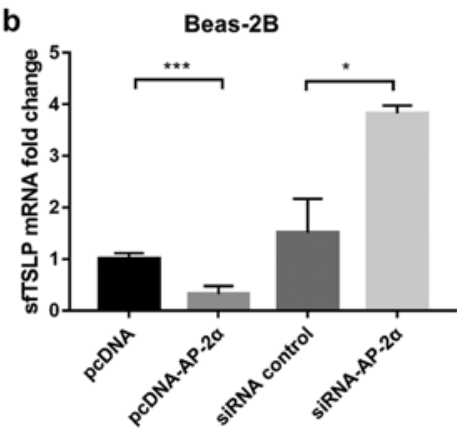

m pcDNA

- pcDNA-AP-2a

- siRNA control

- siRNA-AP-2a

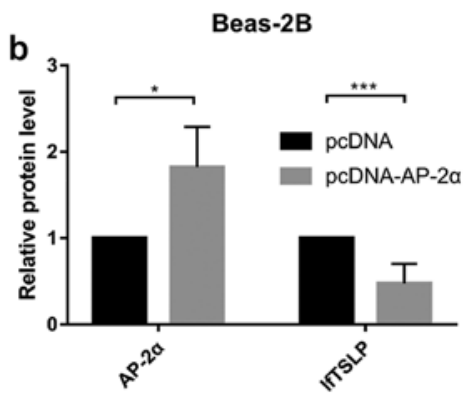

b

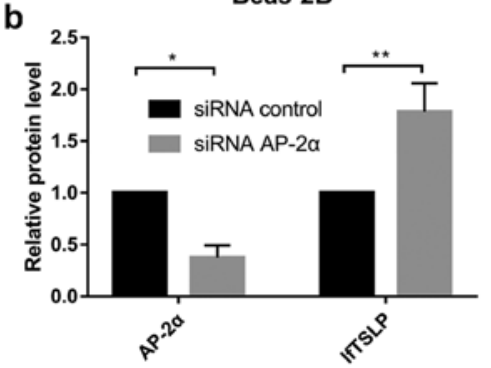

Figure 2. AP-2 $\alpha$ siRNA increases and AP-2 $\alpha$ overexpression decreases lfTSLP and sfTSLP mRNA expression in Beas-2B cells. (A) Beas-2B cells were transfected with AP- $2 \alpha$ overexpression plasmid (pcDNA-AP-2 $\alpha$ ) or siRNA-AP-2 $\alpha$ for $24 \mathrm{~h}$. (a) Quantification of lfTSLP mRNA level after transfection. (b) Quantification of sfTSLP mRNA level after transfection. (c) Quantification of AP-2 $\alpha$ mRNA level after transfection. (Ba) Following transfection with AP- $2 \alpha$ overexpression plasmid (pcDNA-AP- $2 \alpha$ ) or control plasmid (pcDNA) for $48 \mathrm{~h}$, the levels of lfTSLP and AP-2 $\alpha$ proteins were detected in Beas-2B cells by western blotting. (b) Quantification of western blotting. (Ca) Following transfection with AP-2 $\alpha$-siRNA or siRNA control for $48 \mathrm{~h}$, the levels of lfTSLP and AP- $2 \alpha$ proteins were detected in Beas-2B cells by western blotting. (b) Quantification of western blotting. Each experiment was performed in triplicate and significant differences were determined with the Student's t-test. ${ }^{*} \mathrm{P}<0.05,{ }^{* * *} \mathrm{P}<0.01,{ }^{* * * *} \mathrm{P}<0.001,{ }^{* * * * *} \mathrm{P}<0.0001$ vs. the corresponding control. AP-2, activating protein 2; si, small interfering; lf, long isoform; sf, short isoform; TSLP, thymic stromal lymphopoietin.

IfTSLP expression was regulated by AP-2 $\alpha$ in the pulmonary epithelial cells (Fig. 2B and C).

AP-2 $\alpha$ regulates sfTSLP transcriptional promoter activity. To elucidate the specific promoter region responsible for sfTSLP regulation, JASPAR (http://jaspar.genereg.net/analysis) (18) was used to predict the AP-2 $\alpha$ transcriptional sites of the promoter 5'-flanking region. Several putative AP-2 $\alpha$ binding sites were identified. A series of 5'-flanking regions of sfTSLP were inserted to pGL3-Basic as before (Fig. S1A). As shown in Fig. 3A, the core promoter region of sfTSLP was located at $-200 /+25 \mathrm{nt}$ as revealed in our previous experiment (17). A statistically significant decrease $(\sim 60 \%)$ in luciferase activity was observed in response to Ap- $2 \alpha$ overexpression.

To further confirm that the binding site at $-200 /+25 \mathrm{nt}$ (Fig. 3B) is critical for sfTSLP modulation, site-directed mutagenesis targeting $\mathrm{AP}-2 \alpha$ in the context of the core promoter region was performed (Fig. 3C-a). A $76.5 \%$ decrease in the luciferase activity was observed in wild-type pGL3-200/+25 when AP-2 $\alpha$ was overexpressed (Fig. 3C-c) and 
A

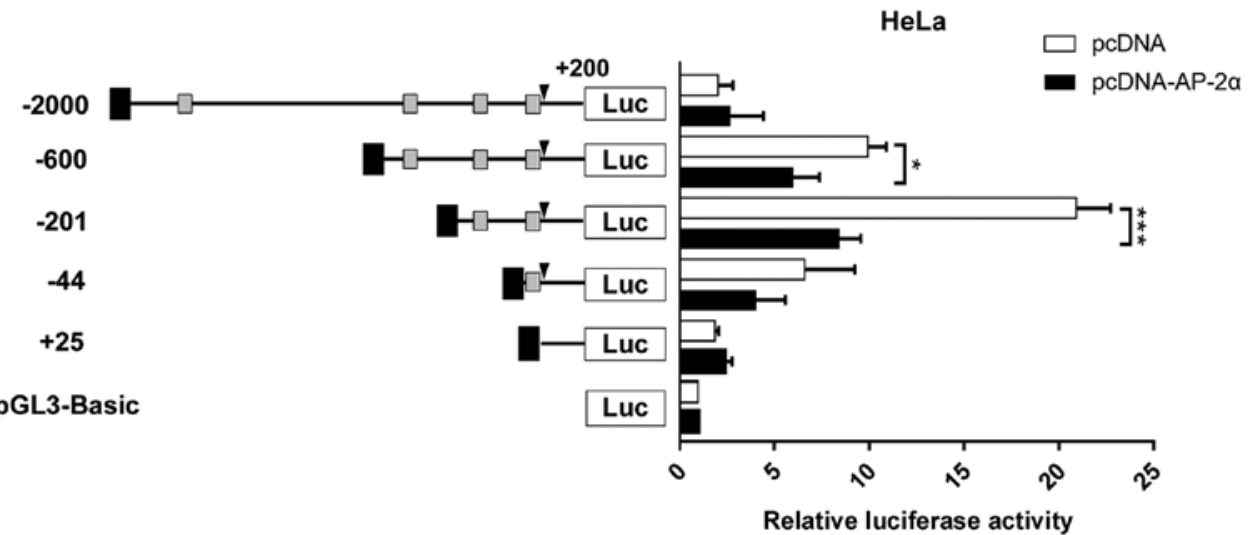

B

-201 TGTTCCAATTTAGGGAGAGGAAGTCTCTATCCGGAGGAAAGGCAAATTGGGAACTGGGACGAGGGAACG -133 TTGTTAGGGGCACCACCTGCTGGGGTCCGGCGCCTCCGCGCTCGGGCTCGGAATTTTGGAGCCTCCGC -64 CCCCTGGAGACTTGGGAGGAGCAGCGTGGGTGACAGTCTTTTCGCGACGAGT GCCCTCCGCFACCCTC +7 GCCACGCCCCTGCTCCCCC

C a

AP-2 $\alpha$ binding site

Mut-AP-2 $\alpha$

GCCстCсGC

GCAATCCGC
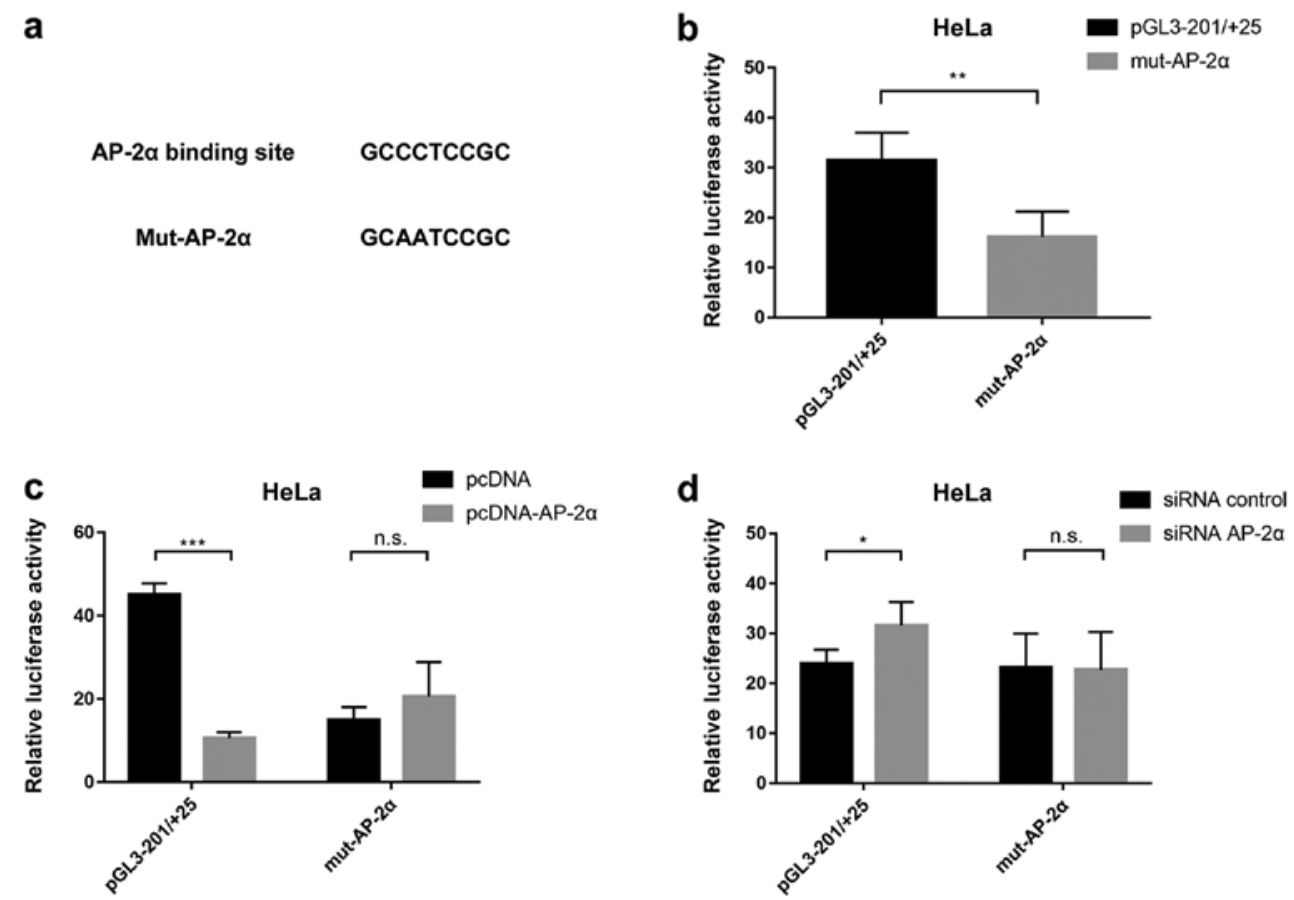

Figure 3. Effect of AP-2 $\alpha$ on sfTSLP promoter-driven luciferase activity. (A) Various 5' deletions of the sfTSLP promoter were cloned in pGL3-Basic and transiently co-transfected with AP- $2 \alpha$ expression plasmid or control plasmid in HeLa cells. The length of each construct is marked. The positions of AP- $\alpha$ binding sites are marked by grey squares and the transcription start site by a black triangle. (B) Nucleotide sequence of the core promoter region of sfTSLP. The putative binding site for AP-2 $\alpha$ is marked with a box and the transcription start site by a dot. (C) Luciferase assay. (a) Nucleotide sequence of the putative binding site for AP- $2 \alpha$ and mutations of the AP- $2 \alpha$ binding sites. (b) Site-directed mutagenesis of AP-2 $\alpha$ were cloned in pGL3-Basic. The plasmids containing the proximal minimal promoter of sfTSLP (pGL3 -200/+25) and mutations of the AP-2 $\alpha$ binding sites on the sfTSLP minimal promoter were constructed and transfected into HeLa cells. (c) The AP-2 $\alpha$ expression plasmid or control vector was co-transfected into HeLa cells together with 100 ng of luciferase reporter plasmid driven by wild-type or mutated (mut) sfTSLP promoter. (d) The siRNA AP-2 $\alpha$ or siRNA control was co-transfected into HeLa cells together with $100 \mathrm{ng}$ of luciferase reporter plasmid driven by wild-type or mutated sfTSLP promoter. Student's t-test was performed. ${ }^{*} \mathrm{P}<0.05,{ }^{* *} \mathrm{P}<0.01,{ }^{* * *} \mathrm{P}<0.001 ; \mathrm{n} . \mathrm{s}$. not significant. Results are shown as mean \pm standard deviation of representative data of $\geq 3$ independent experiments. AP- $2 \alpha$, activating protein $2 \alpha$; sf, short isoform; TSLP, thymic stromal lymphopoietin.

a $31.9 \%$ increase when AP-2 $\alpha$ was knocked down by siRNA (Fig. 3C-d). By contrast, the mutant plasmid did not alter the AP-2 $\alpha$-mediated TSLP promoter activity. The activity of reporter genes was significantly reduced (by $\sim 50 \%$ ) after the -10/-2 nt AP-2 $\alpha$ site mutation, compared with that in wild-type pGL3 -200/+25 (Fig. 3C-b). Therefore, the imperfect AP-2 $\alpha$ site at $-102 /-94$ nt was mutated (Fig. S1A) and a 50\% increase in luciferase activity in wild-type was observed (Fig. S1B). A decrease of $25 \%$ was observed after both AP- $2 \alpha$ binding sites were mutated (Fig. S1B). The luciferase activity of plasmid with the mutation of at the two AP- $2 \alpha$ sites was significantly different from that in the mutation of AP-2 $\alpha$ site at -102/-94 nt $(\mathrm{P}<0.05)$, but not from that in the mutation of AP- $2 \alpha$ site at $-10 /-2$ nt. This meant that the main binding site was located 

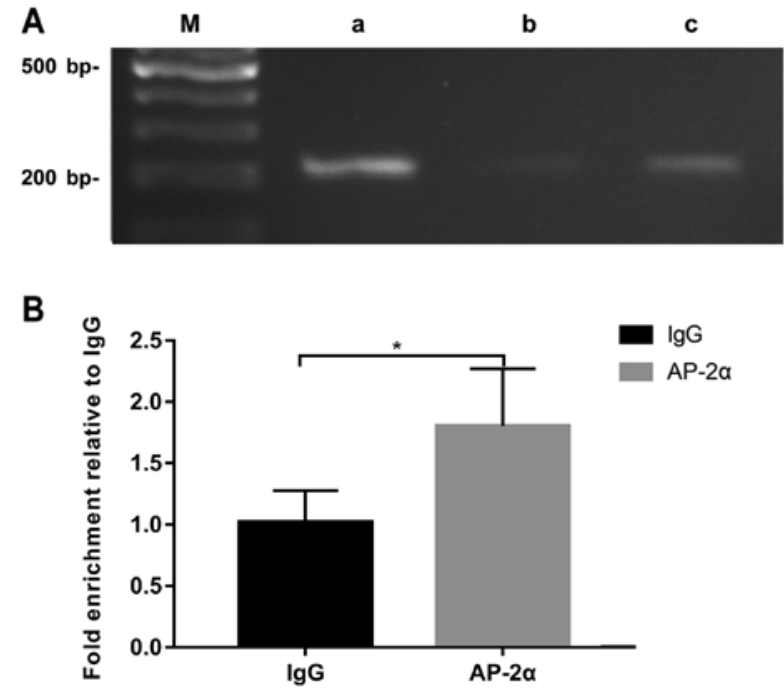

Figure 4. AP- $2 \alpha$ binds to the AP- $2 \alpha$ motif of the human sfTSLP promoter in vivo. (A) The chromatin fragments were PCR-amplified using primers specific to the proximal AP- $2 \alpha$ binding site of the sfTSLP promoter and resolved on an agarose gel. Input DNA was used as a positive control and no-specific rabbit IgG as a negative control during PCR. M, 100-bp DNA ladder; a, positive control; $b$, negative control and c, anti-AP-2 $\alpha$. (B) Chromatin fragments were quantified by reverse transcription-quantitative PCR. Data are expressed as the fold change in relative expression of antibodies to normal IgG controls Representative data of 3 experiments. ${ }^{*} \mathrm{P}<0.05$. AP- $2 \alpha$, activating protein $2 \alpha$; sf, short isoform; TSLP, thymic stromal lymphopoietin.

at $-10 /-2$ nt. These results pointed to the important function of AP-2 $\alpha$ site at $-200 /+25$ nt in sfTSLP expression.

AP-2 $\alpha$ directly binds to the sfTSLP promoter in vivo. ChIP assays were performed in Beas-2B cells to determine whether AP- $2 \alpha$ could directly or indirectly regulate sfTSLP expression in vivo. The ultrasonic extract of Beas-2B cells cross-linked with formaldehyde was immunoprecipitated with the anti-AP-2 $\alpha$ antibody. PCR was performed based on the relevant chromatin DNA fragments, using primers flanking the AP- $2 \alpha$ binding site in the core promoter region. The reactions with anti-histone $\mathrm{H} 3$ antibodies were used as positive controls, while those of anti-IgG were used as negative controls. As shown in Fig. 4A, the anti-AP- $2 \alpha$ antibody was more abundant in the Beas-2B cells than in the AP- $2 \alpha$ promoter precipitated by the $\mathrm{IgG}$ antibody. The DNA fragments were further quantified by RT-qPCR (Fig. 4B). In conclusion, the present study suggested that AP- $2 \alpha$ could directly bind to the core promoter region of sfTSLP.

\section{Discussion}

It has been reported that sfTSLP/lfTSLP ratio changes in certain diseases, such as gut disorders, atopic dermatitis and skin keratosis exposed to inflammatory stimuli $(6,19)$. In viral respiratory infections, the aberrant expression of long isoform thymic stromal lymphopoietin (IfTSLP) triggers Th2 overreaction and pathological exacerbation, eventually resulting in asthma (20,21), while the role of short isoform TSLP (sfTSLP) in airway epithelial cells attacked by respiratory viruses is still unclear. Previous research has demonstrated that sfTSLP is almost uninduced by proinflammatory cytokines, such as poly(I:C), lipopolysaccharide, or macrophage-activating lipopeptide 2 (6) and appears to be downregulated in inflammation (22). However, the expression of sfTSLP mRNA increases in bronchial epithelial cell lines challenged with human pneumovirus (23). To elucidate the underlying mechanism, the present study explored the differential expression of lfTSLP and sfTSLP in RSV infection. It has been confirmed that IfTSLP is secreted by RSV-attached airway epithelial cells $(13,24)$, but the role of sfTSLP has been rarely reported. The present study detected the expression profiles of both isoforms in Beas-2B cells following RSV infection. Polyclonal antibodies, with a previously verified feasibility, were used to detect the protein level of lfTSLP $(6,19,22)$. Consistent with the findings of previous studies $(20,21)$, lfTSLP showed a significant increase at the mRNA and protein levels. As the sfTSLP protein sequence overlaps the IfTSLP sequence in the C-terminal region, it was not possible to distinguish the expression of sfTSLP at the protein level, but a significant increase was observed at the mRNA level.

The present study further investigated the potential transcriptional pathways mediating RSV-induced TSLP expression in Beas-2B cells. It has been reported that pro-inflammatory cytokines can induce lfTSLP by a transcriptional mechanism regulated by nuclear factor (NF) $\mathrm{\kappa B}$ and transcription factor AP-1 (25-27). However, sfTSLP is not sensitive to proinflammatory cytokines $(6,28)$, meaning that the activation of $N F-\kappa B$ and AP-1 is critical for inflammation-induced expression of lfTSLP, but not sfTSLP.

AP- $2 \alpha$ is a transcription factor that regulates the proliferation and differentiation of mammalian cells (29). It has been reported that AP-2 $\alpha$ can upregulate Toll-like receptor 2 (TLR2) gene expression, and expression of lfTSLP is induced through the TLR2-TLR6 pathway in human monocyte cell lines $(30,31)$. It has also been reported that the reduced AP- $2 \alpha$ protein binds to TSLP promoter polymorphism reference SNP rs2289276 site to increase a child's susceptibility to atopic asthma (13). However, no functional studies have been carried out. In the present study, limited AP-2 $\alpha$ expression was observed in RSV-infected Beas-2B cells. It was first hypothesized that the increase in sfTSLP expression was caused by a decrease in AP-2 $\alpha$ expression, since sfTSLP cannot be induced by NF- $\mathrm{B}$ and AP-1. Further experiments showed that the overexpression of AP- $2 \alpha$ reduced the promoter activity, mRNA levels of sfTSLP and the mRNA and protein levels of IfTSLP, while inhibition of AP-2 $\alpha$ expression increased sfTSLP and IfTSLP expression. Transcriptional activation on $5^{\prime}$ deletion of the human IfTSLP promoter has been studied in airway epithelial cells (27). Harada et al (13) showed that AP-2 $\alpha$ directly binds to the promoter of 1fTSLP and may act as a transcription-suppressing factor. However, no studies, to the best of the authors' knowledge, have investigated the association between AP- $2 \alpha$ and sfTSLP transcriptional promoter activity. In the present study, AP- $2 \alpha$ was identified as an important regulator in sfTSLP expression in Beas-2B cells and the AP- $2 \alpha$ binding site in the core promoter region of sfTSLP was shown to be critical for sfTSLP modulation. Notably, the mutation of the imperfect binding site increased the luciferase activity of sfTSLP core promoter, but the mutation of the main AP- $2 \alpha$ site located at $-10 /-2 n t$ significantly reduced the luciferase activity $(\mathrm{P}<0.05)$. This meant that the activity of reporter genes was 
weakened, instead of increased (as expected), following the mutation of the main AP- $2 \alpha$ site. Adjacent to the transcription initiation site, AP- $2 \alpha$ may compete to inhibit certain positive or general transcription factors (32), including general transcription factor IIB (33) and influence the formation of pre-initiation complex. These observations indicate that AP- $2 \alpha$ is a transcription-suppressing factor of both IfTSLP and sfTSLP.

SfTSLP is a homeostatic and anti-inflammatory isoform expressed in steady-state. By contrast, lfTSLP is pro-inflammatory and only expressed during inflammatory processes $(6,19)$. LfTSLP-induced inflammation can be partially reversed by sfTSLP, especially in HDM-induced asthmatic airway epithelial cells (34). SfTSLP can condition both mature dendritic cells and monocyte-derived dendritic cells and limit their inflammatory potential on monocyte-derived dendritic cells (19). The reduction of interferon (IFN) $\lambda$ is also associated with sfTSLP. IFN $\lambda$ is also anti-inflammatory in that it enhances adaptive mucosal immunity by promoting the release of TSLP (35). The increase of sfTSLP in RSV infection could counter inflammation, especially lfTSLP-induced inflammation. However, the interaction between sfTSLP and lfTSLP in RSV infection should be confirmed by further functional studies.

In the present study, higher TSLP production appeared with the reduction of AP- $2 \alpha$ in bronchial epithelial cells undergoing viral respiratory infections. It has been demonstrated that lfTSLP is increased in RSV infection (19-21). To the best of the authors' knowledge, this is the first study to investigate the expression of sfTSLP in RSV infection in bronchial epithelial cells. Previous studies have mentioned the possible association between 1fTSLP and AP-2 $\alpha$ : AP- $2 \alpha$ binds to the IfTSLP promoter and this binding is inhibited in inflammation to exert a protective effect $(13,27)$. However, this is a conjecture that has not been verified by experiments. The present study showed that AP- $2 \alpha$ regulated lfTSLP expression at both mRNA and protein levels. The present study also showed that AP- $2 \alpha$ regulated SfTSLP expression and that AP- $2 \alpha$ directly bound to sfTSLP promoter. It also identified the transcription of sfTSLP in bronchial epithelial cells and the association between sfTSLP and AP-2 $\alpha$. Further studies should be performed to uncover the molecular mechanisms by which sfTSLP expression regulates RSV infection, cellular function and disease pathophysiology.

\section{Acknowledgements}

Not applicable.

\section{Funding}

This work was supported by grants from the National Natural Science Foundation of China (grant no. 81970579 to GPZ), Jiangsu Province Science and Education Enhancing Health Project Innovation Team (Leading Talent) Program (grant no. CXTDA2017018 to GPZ) and A Project Funded by the Priority Academic Program Development of Jiangsu Higher Education Institutions.

\section{Availability of data and materials}

The analyzed datasets generated during the study are available from the corresponding author on reasonable request.

\section{Authors' contributions}

JH, QC and GPZ made substantial contributions to the conception and design of the study. JH carried out most of the experiments and wrote the manuscript. QC provided suggestions for the first draft. DDF and JHC participated in data acquisition, analysis and interpretation. All authors read and approved the final manuscript.

\section{Ethics approval and consent to participate}

Not applicable.

\section{Patient consent for publication}

Not applicable.

\section{Competing interests}

The authors declare that they have no competing interests.

\section{References}

1. Malinczak CA, Fonseca W, Rasky AJ, Ptaschinski C, Morris S, Ziegler SF and Lukacs NW: Sex-associated TSLP-induced immune alterations following early-life RSV infection leads to enhanced allergic disease. Mucosal Immunol 12: 969-979, 2019.

2. Lee HC, Headley MB, Loo YM, Berlin A, Gale M Jr, Debley JS, Lukacs NW and Ziegler SF: Thymic stromal lymphopoietin is induced by respiratory syncytial virus-infected airway epithelial cells and promotes a type 2 response to infection. J Allergy Clin Immunol 130: 1187-1196.e5, 2012.

3. Lin SC, Cheng FY, Liu JJ and Ye YL: Expression and regulation of thymic stromal lymphopoietin and thymic stromal lymphopoietin receptor heterocomplex in the innate-adaptive immunity of pediatric asthma. Int J Mol Sci 19: 1231, 2018.

4. Martin Mena A, Langlois A, Speca S, Schneider L, Desreumaux P, Dubuquoy L and Bertin B: The Expression of the short isoform of thymic stromal lymphopoietin in the colon is regulated by the nuclear receptor peroxisome proliferator activated receptor-gamma and is impaired during ulcerative colitis. Front Immunol 8: 1052, 2017.

5. Wilson SR, Thé L, Batia LM, Beattie K, Katibah GE, McClain SP, Pellegrino M, Estandian DM and Bautista DM: The epithelial cell-derived atopic dermatitis cytokine TSLP activates neurons to induce itch. Cell 155: 285-295, 2013.

6. Bjerkan L, Schreurs O, Engen SA, Jahnsen FL, Baekkevold ES, Blix IJ and Schenck K: The short form of TSLP is constitutively translated in human keratinocytes and has characteristics of an antimicrobial peptide. Mucosal Immunol 8: 49-56, 2015.

7. Jartti T and Gern JE: Role of viral infections in the development and exacerbation of asthma in children. J Allergy Clin Immunol 140: 895-906, 2017.

8. Rossi GA and Colin AA: Infantile respiratory syncytial virus and human rhinovirus infections: Respective role in inception and persistence of wheezing. Eur Respir J 45: 774-789, 2015.

9. Dumas O, Hasegawa K, Mansbach JM, Sullivan AF, Piedra PA and Camargo CA Jr: Severe bronchiolitis profiles and risk of recurrent wheeze by age 3 years. J Allergy Clin Immunol 143: 1371-1379.e7, 2019.

10. Coultas JA, Smyth R and Openshaw PJ: Respiratory syncytial virus (RSV): A scourge from infancy to old age. Thorax 74: 986-993, 2019.

11. Feldman AS, He Y, Moore ML, Hershenson MB and Hartert TV: Toward primary prevention of asthma. Reviewing the evidence for early-life respiratory viral infections as modifiable risk factors to prevent childhood asthma. Am J Respir Crit Care Med 191: 34-44, 2015.

12. Qiao J, Li A and Jin X: TSLP from RSV-stimulated rat airway epithelial cells activates myeloid dendritic cells. Immunol Cell Biol 89: 231-238, 2011. 
13. Harada M, Hirota T, Jodo AI, Hitomi Y, Sakashita M, Tsunoda T, Miyagawa T, Doi S, Kameda M, Fujita K, et al: Thymic stromal lymphopoietin gene promoter polymorphisms are associated with susceptibility to bronchial asthma. Am J Respir Cell Mol Biol 44: 787-793, 2011.

14. Wang XH, Shu J, Jiang CM, Zhuang LL, Yang WX, Zhang HW, Wang LL, Li L, Chen XQ, Jin R, et al: Mechanisms and roles by which IRF-3 mediates the regulation of ORMDL3 transcription in respiratory syncytial virus infection. Int $\mathrm{J}$ Biochem Cell Biol 87: 8-17, 2017.

15. Reed LJ and Muench H: A simple method of estimating fifty percent endpoint. Am J Hyg 27: 493-497, 1938.

16. Livak KJ and Schmittgen TD: Analysis of relative gene expression data using real-time quantitative PCR and the $2(-\Delta \Delta \mathrm{C}(\mathrm{T}))$ Method. Methods 25: 402-408, 2001.

17. He J, Cao Q and Zhou GP: Identification and characterization of human short isoform TSLP promoter. J Nanjing Med Univ 40: 10-14, 2020 (In Chinese).

18. Fornes O, Castro-Mondragon JA, Khan A, van der Lee R, Zhang X, Richmond PA, Modi BP, Correard S, Gheorghe M, Baranašić D, et al: JASPAR 2020: Update of the open-access database of transcription factor binding profiles. Nucleic Acids Res 48: D87-D92, 2020.

19. Fornasa G, Tsilingiri K, Caprioli F, Botti F, Mapelli M, Meller S, Kislat A, Homey B, Di Sabatino A, Sonzogni A, et al: Dichotomy of short and long thymic stromal lymphopoietin isoforms in inflammatory disorders of the bowel and skin. J Allergy Clin Immunol 136: 413-422, 2015.

20. Vázquez Y, González L, Noguera L, González PA, Riedel CA, Bertrand P and Bueno SM: Cytokines in the respiratory airway as biomarkers of severity and prognosis for respiratory syncytial virus infection: An update. Front Immunol 10: 1154-1154, 2019.

21. Stier MT, Bloodworth MH, Toki S, Newcomb DC, Goleniewska K, Boyd KL, Quitalig M, Hotard AL, Moore ML, Hartert TV, et al: Respiratory syncytial virus infection activates IL-13-producing group 2 innate lymphoid cells through thymic stromal lymphopoietin. J Allergy Clin Immunol 138: 814-824.e11, 2016.

22. Bjerkan L, Sonesson A and Schenck K: Multiple functions of the new cytokine-based antimicrobial peptide thymic stromal lymphopoietin (TSLP). Pharmaceuticals (Basel) 9: 41, 2016.

23. Li Y, Lund C, Nervik I, Loevenich S, Døllner H, Anthonsen MW and Johnsen IB: Characterization of signaling pathways regulating the expression of pro-inflammatory long form thymic stromal lymphopoietin upon human metapneumovirus infection Sci Rep 8: 883, 2018.

24. Zhao Y, Jamaluddin M, Zhang Y, Sun H, Ivanciuc T, Garofalo RP and Brasier AR: Systematic analysis of cell-type differences in the epithelial secretome reveals insights into the pathogenesis of respiratory syncytial virus-induced lower respiratory tract infections. J Immunol 198: 3345-3364, 2017.
25. Redhu NS, Saleh A,Halayko AJ, Ali AS and Gounni AS: Essential role of NF- $\kappa$ B and AP- 1 transcription factors in TNF- $\alpha$-induced TSLP expression in human airway smooth muscle cells. Am J Physiol Lung Cell Mol Physiol 300: L479-L485, 2011.

26. Cultrone A, de Wouters T, Lakhdari O, Kelly D, Mulder I, Logan E, Lapaque $\mathrm{N}$, Doré $\mathrm{J}$ and Blottière HM: The NF- $\kappa \mathrm{B}$ binding site located in the proximal region of the TSLP promoter is critical for TSLP modulation in human intestinal epithelial cells. Eur J Immunol 43: 1053-1062, 2013.

27. Lee H-C and Ziegler SF: Inducible expression of the proallergic cytokine thymic stromal lymphopoietin in airway epithelial cells is controlled by NFkappaB. Proc Natl Acad Sci USA 104: 914-919, 2007.

28. Harada M, Hirota T, Jodo AI, Doi S, Kameda M, Fujita K, Miyatake A,Enomoto T, Noguchi E, Yoshihara S, et al: Functional analysis of the thymic stromal lymphopoietin variants in human bronchial epithelial cells. Am J Respir Cell Mol Biol 40: 368-374, 2009.

29. Eckert D, Buhl S, Weber S, Jäger R and Schorle H: The AP-2 family of transcription factors. Genome Biol 6: 246, 2005.

30. Vu AT, Baba T, Chen X, Le TA, Kinoshita H, Xie Y, Kamijo S, Hiramatsu K, Ikeda S, Ogawa H, et al: Staphylococcus aureus membrane and diacylated lipopeptide induce thymic stromal lymphopoietin in keratinocytes through the Toll-like receptor 2-Toll-like receptor 6 pathway. J Allergy Clin Immunol 126 : 985-993, 993.e1-993.e3, 2010

31. Li M, Li X, Wang E and Luo E: Upregulation of Toll-like receptor 2 gene expression by acetylation of AP-2 alpha in THP-1 cells, a human monocytic cell line. Int J Biochem Cell Biol 45: 1594-1599, 2013.

32. Haberle V and Stark A: Eukaryotic core promoters and the functional basis of transcription initiation. Nat Rev Mol Cell Biol 19: 621-637, 2018

33. Lagrange T, Kapanidis AN, Tang H, Reinberg D and Ebright RH: New core promoter element in RNA polymerase II-dependent transcription: Sequence-specific DNA binding by transcription factor IIB. Genes Dev 12: 34-44, 1998.

34. Dong H, Hu Y, Liu L, Zou M, Huang C, Luo L, Yu C, Wan X, Zhao H, Chen J, et al: Distinct roles of short and long thymic stromal lymphopoietin isoforms in house dust mite-induced asthmatic airway epithelial barrier disruption. Sci Rep 6: 39559, 2016.

35. Ye L, Schnepf D, Becker J, Ebert K, Tanriver Y, Bernasconi V, Gad HH, Hartmann R, Lycke $N$ and Staeheli P: Interferon- $\lambda$ enhances adaptive mucosal immunity by boosting release of thymic stromal lymphopoietin. Nat Immunol 20: 593-601, 2019 